Instrumental Achievements

\title{
Laboratory Automation System for the Analysis of Drugs in Biological Fluids
}

\author{
Kiyoshi BanNo* and Reiji TAKAHASHI** \\ *Analytical Chemistry Research Laboratory, Tanabe Seiyaku Co., Ltd., \\ Kashima, Yodogawa, Osaka 532, Japan \\ **Department of Chemistry, Ritsumeikan University, Toujïnkita, Kita, Kyoto 603, Japan
}

\begin{abstract}
Keywords Laboratory automation, robotic automation, local area network, data processing system, biological fluid, drug
\end{abstract}

Recently we have reported a data processing system for stability studies of new drugs. ${ }^{1-3}$ Since the commercial introduction of the first laboratory robot by Zymark $\mathrm{Co}$. in 1982, robotics have been applied to pharmaceutical analysis ${ }^{4,5}$, dissolution tests ${ }^{6}$ and many other assay types. A flexible system for the analysis of drugs in biological samples was reported by Fouda and Schneider ${ }^{7}$ in 1987. But this system involving filtration and liquid-solid extraction process has not been constructed.

In the present work, we have developed a laboratory automation system which is composed of a laboratory robot for the sample preparations, a micro-high-performance liquid chromatograph and personal computers for the data processing.

\section{Experimental}

\section{Components of the laboratory automation system}

The system configuration is schematically shown in Fig. 1. The core of the system is the Zymate robot which includes the arm and the robot controller. The robot controller is the central processing unit in which all the experimental steps are programmed. The microhigh-performance liquid chromatograph (HPLC) equipped with a BAS PM-60 pump unit (West Lafayette, USA) and the UV spectrophotometer was a Shimadzu SPD-6A type. The digital diluter pipetter used was a Shimadzu DIP-1 type (Kyoto, Japan). The ultrasonic bath was a Branson B-12 model. The evaporator was a Pierce Reacti-Therm heating module (Rockford, USA). The controller allows the operator and the program to call commands or collect status information from various modules. Figure 1 indicates the direction of communication among various system components.

The experimental data can be compiled and printed at the time of measurement by using a local area network system. The local area network system was systematized using four central file-server computers (NEC PC9801 XLW-type, Tokyo, Japan) each equipped with
$40 \mathrm{MB}$ of main memory.

\section{Adaptation}

The robotic system includes functions for pipetting a constant volume of biological fluids and performs dilution, weighing of sample, capping the test tubes, mixing, solvent extraction, solid extraction, filtration, centrifugation, evaporation, reconstitution and injection into a micro-HPLC system.

The master laboratory station is the source of all volumetric measurements and all liquid transfers about the robot working area. The ultrasonic bath was used for dissolution of the reference standard materials and for mixing of solution. The vortex mixer was used for liquid-liquid extraction of drugs from biological samples. In the liquid-solid extraction, the technique involves the use of disposable columns of $1 \mathrm{ml}$ or $3 \mathrm{ml}$ capacity. These columns are pre-packed with one of a number of different materials with varying adsorption characteristics. Contaminating substances in the biological fluids are removed by washing the columns with various solvents. Then the organic solvent is added to the column to elute the drugs of interest. The evaporator, for the evaporation of solvent extracts with nitrogen gas and heat, was modified for control by the Zymate program. Constant volume of the internal standard solution was added by the digital diluter pipetter. Sample solutions are aspirated through a sample tip into a fixed volume injection loop using the syringe. The measurement conditions of HPLC are controlled by the computer.

The signal from the detector is sent to the interface and is transferred to a local computer; then, it is stored on a hard disk in a local area network (LAN) system.

\section{Application}

The laboratory automation system which includes the robotic system was applied to prepare the test solutions of some drugs from plasma samples. This robotic system performs the following 10 steps in $40 \mathrm{~min}$ before 


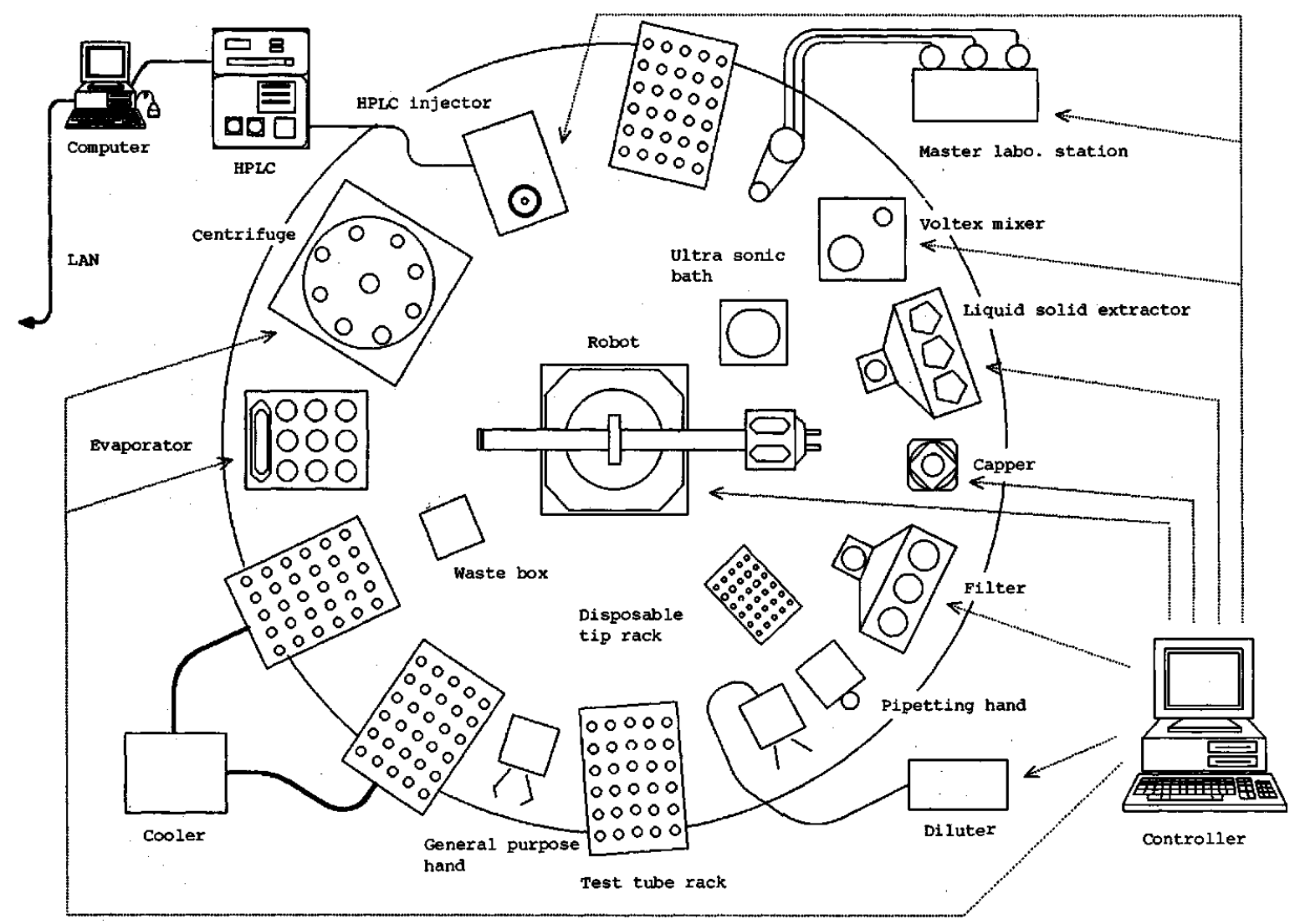

Fig. 1 Schematic diagram of the laboratory automation system for the analysis of drugs in biological fluids.

HPLC injection. HPLC proceeding time requires $90 \mathrm{~min}$. 1) dissolve standard materials, 2) dilute with ethanol, 3) take $1 \mathrm{ml}$ of plasma and add $0.1 \mathrm{ml}$ of internal standard solution, 4) apply $1.1 \mathrm{ml}$ of plasma to C18, solid-liquid extraction column, 5) wash with $1 \mathrm{ml}$ of $0.1 \mathrm{M}$ hydrochloric acid and methanol, 6) elute with $80 \%$ acidic ethanol, 7) centrifuge at $1800 \mathrm{~g}$ for $5 \mathrm{~min}, 8$ ) take $0.5 \mathrm{ml}$ of eluate, 9) evaporate under a stream of nitrogen gas at $30^{\circ} \mathrm{C}, 10$ ) add $0.2 \mathrm{ml}$ of mobile phase, 11) inject into HPLC, 12) send the HPLC data to the computers in a LAN system.

\section{Results and Discussion}

In order to ensure the reproducibility of the pipetting step, adding step and fractionating step, various volumes pipetted with the master laboratory station were compared with manually pipetted volumes. The syringe drew up $0.1 \mathrm{ml}$ of the solution, but some of the liquid dripped out of the pipet tip before it could be added to the extraction column. This problem was solved by drawing up $0.02 \mathrm{ml}$ of air into the pipet tip after the solution was drawn up.

The results of reproducibility in each step are shown in Table 1. Relative standard deviations (RSD) of the weights of dispensed volumes using the robotic system
Table 1 Comparison between robotic and manual procedures

\begin{tabular}{lccc}
\hline \multirow{2}{*}{ Preparation method } & \multirow{2}{*}{ Amount } & \multicolumn{2}{c}{ RSD, \% $(n=10)$} \\
\cline { 3 - 4 } & & Robotic & Manual \\
\hline Pipetting step & $0.5 \mathrm{ml}$ & 0.6 & 1.1 \\
& $1.0 \mathrm{ml}$ & 0.4 & 1.0 \\
Adding step & $0.5 \mathrm{ml}$ & 0.3 & 1.1 \\
& $6.0 \mathrm{ml}$ & 0.1 & 0.1 \\
Fractionating step & $1.0 \mathrm{ml}$ & 0.8 & 1.0 \\
& $5.0 \mathrm{ml}$ & 0.4 & 0.4 \\
\hline
\end{tabular}

Table 2 Comparison of reproducibility of HPLC analyses using automatic system and manual methods

\begin{tabular}{ccc}
\hline \multirow{2}{*}{$\begin{array}{c}\text { Concentration } \\
(\mathrm{ng} / \mathrm{ml}, \text { plasma })\end{array}$} & \multicolumn{2}{c}{$\mathrm{RSD}, \%,(n=5)$} \\
\cline { 2 - 3 } & Automation system & Manual \\
\hline 5 & 8.6 & 9.5 \\
10 & 8.1 & 7.9 \\
50 & 5.3 & 6.7 \\
\hline
\end{tabular}

were consistently less than those obtained manually.

Reproducibility values of HPLC analysis at low levels of a drug in plasma using the robotics and manual 
method are listed in Table 2. The instrumental analysis was performed by the internal standard method. As shown in Table 2, the robotic method demonstrated reproducibility superior to that of the manual method. Chromatograms obtained with the robotic methods were almost the same as those from the manual methods.

On the other hand, in the manual procedure, centrifugation is used to force liquids through the extraction columns, whereas the robotic procedure uses air pressure. The essential similarity of the two procedures allows a direct comparison between automated and manual extraction techniques.

The robot can be programmed to run one extraction procedure and then switch to another, since the analytical time period is longer than the time required to extract a sample. The present robotic system has demonstrated sufficient accuracy, precision and reliability to perform a drug analysis.

The experimental data from the analytical instruments are sent to the personal computers in the LAN system, which record all analytical data on the data base, and make data references and prints easily available. The LAN system improved the working rate and precision of measurement instruments, since possible errors which might have occurred in calculation and data recording are eliminated by connecting the computers.

The authors thank Dr. Toshio Kakimoto, General Manager of our laboratory, for suggestions and discussion.

\section{References}

1. R. Shimizu, M. Matsuo, T. Miyamoto, Y. Shimaoka, H. Mano, K. Banno and Y. Fujikawa, "Advances in Laboratory Automation Robotics", ed. Janet R. Strimaitis and Gerald L. Hawk, Vol. 2, p. 733, Zymark Corporation, Hopkinton, 1985.

2. R. Shimizu, Y. Shimaoka, S. Muraki, H. Mano and K. Banno, Iyakuhin Kenkyu, 19, 74 (1988).

3. K. Banno, S. Muraki, H. Mano, J. Kato and T. Kakimoto, Lyakuhin Kenkyu, 21, 430 (1990).

4. S. A. Borman, Anal Chem., 57, 651 a (1985).

5. J. H. Johnson, R. Srinival and T. J. Kinzelman, Am. Lab., 9, 50 (1985).

6. K. Banno, S. Muraki, H. Mano, H. Nakai and T. Kakimoto, Iyakuhin Kenkyu, 22, 25 (1991).

7. H. G. Fouda and R. P. Schneider, Tr. Anal. Chem., 6, 139 (1987).

(Received February 1, 1991)

(Accepted March 5, 1991) 